\title{
Minimizing Total Weighted Completion Time on Single Machine with Past-Sequence-Dependent Setup Times and Exponential Time-Dependent and Position-Dependent Learning Effects
}

\author{
Kaibiao Sun and Hongxing Li \\ School of Electronic and Information Engineering, Dalian University of Technology, Dalian 116024, China \\ Correspondence should be addressed to Hongxing Li, lihx-lab@163.com
}

Received 13 May 2009; Revised 29 August 2009; Accepted 17 September 2009

Recommended by Stevo Stević

This paper addresses a single-machine problem in which the past-sequence-dependent (p-s-d) setup times and exponential time-dependent and position-dependent learning effects are considered. By the exponential time-dependent learning effect, it means that the processing time of a job is defined by an exponent function of the total actual processing time of the already processed jobs. The setup times are proportional to the length of the already processed jobs. The aim is to minimize the total weighted completion time, this is an NP-hard problem. Under certain conditions, it is shown that the classical WSPT rule is optimal for the problem.

Copyright (C) 2009 K. Sun and H. Li. This is an open access article distributed under the Creative Commons Attribution License, which permits unrestricted use, distribution, and reproduction in any medium, provided the original work is properly cited.

\section{Introduction}

In classical scheduling problems, the processing time of a job is assumed to be a constant. However, in many realistic problems of operations management, both machines and workers can improve their performance by repeating the production operations. Therefore, the actual processing time of a job is shorter if it is scheduled later in a sequence. This phenomenon is known as the "learning effect" in literature (see [1]). During the last few years, learning effect has attracted growing attention from the scheduling research community on account of its significance. Concerning the learning effects, two different approaches in scheduling environments have been suggested. The first one can be best described as position-dependent learning, which was first introduced into the field of scheduling by Biskup in [2] and Cheng and Wang in [3], meaning that learning is affected by the pure number of jobs being processed. Many references have studied such a learning effect model thereafter, a sample of these papers can be seen in [4-13]. However, the position-dependent learning effects neglect the processing times of the jobs already processed. If human interactions 
have a significant impact during the processing of jobs, the processing time will add to the employees' experience and cause learning effects. For situations like this, Kuo and Yang in [14] introduced a time-dependent learning effect, that is, the sum of processing time dependent learning, and showed that the shortest processing time (SPT) rule is optimal for the objective of minimizing the total completion time. For more papers about this timedependent learning model, the reader is referred to the papers [15-17]. Besides, Koulamas and Kyparisis in [18] introduced a general sum of processing time-dependent learning effect model in which employees learn more if they perform a job with a longer processing time. Recently, Wu and Lee in [19] proposed a new learning model where the actual job processing time not only depends on its scheduled position but also depends on the sum of the processing times of jobs already processed. Let $p_{j r}$ denote the actual processing time job $J_{j}$ scheduled in $r$ th position, then $p_{j r}=\left(1+\sum_{k=1}^{r-1} p_{[k]} / \sum_{k=1}^{n} p_{k}\right)^{a_{1}} r^{a_{2}}$, where $p_{[k]}$ is the normal processing time of the $k$ th job in a sequence and $a_{1} \leq 0, a_{2} \leq 0$. Under this model they showed that the SPT sequence is optimal for minimizing makespan and total completion time on single machine under the proposed learning model. In addition, they showed that the WSPT sequence is optimal for minimizing the sum of the weighted completion times if jobs have agreeable weights. Cheng et al. in [20] consider another learning model, in which the learning effect is given as $p_{j r}=\left(1-\sum_{k=1}^{r-1} p_{[k]} / \sum_{k=1}^{n} p_{k}\right)^{a_{1}} r^{a_{2}}$, where $a_{1} \geq 1, a_{2} \leq 0$. Under this learning model, they obtained the same results as that in [19]. Further, they showed that the EDD sequence is optimal for the maximum lateness if jobs have agreeable due dates. For a recent state-of-the-art review on scheduling models and problems with learning effects, the readers are referred to [21].

On the other hand, it is reasonable and necessary to consider scheduling problems with setup times (see [22]). There are two types of setup time or setup cost, namely, sequenceindependent and sequence-dependent. In the first case, the setup time/cost depends solely on the task to be processed, regardless of its preceding task. While in the sequence-dependent type, setup time/cost depends on both the task and its preceding task. For recent results and trends in scheduling problems with setup times or costs, the reader may refer to the recent review paper [23]. Koulamas and Kyparisis in [24] first introduced a scheduling problem with past-sequence-dependent (p-s-d) setup times, that is, the setup time is dependent on all already scheduled jobs. They showed that the standard single-machine scheduling problems with p-s-d setup times to minimize makespan, total completion time and the total absolute differences in completion times are polynomially solvable. They also extended their results to nonlinear p-s-d setup times. Subsequently, Kuo and Yang in [25], Wang in [26], Biskup and Herrmann [27], Wang et al. in [28] studied different single-machine scheduling problems with time-dependent learning effect and setup times considerations.

From the learning models mentioned earlier, it is not hard to see that the actual processing time of, say, the $r$ th job is affected by the sum of the normal processing times of the preceding $r$-1 jobs. This learning effect model is based on the assumption that the number of operating processes in a job depends on the repetitions of the same operation. However, in many situations, the operating processes of a job are different, for example, car repair or maintenance and patient diagnosis and treatment. The conditions of cars or patients are different. Hence, there are no identical repetitions of operating processes in the job. Nevertheless, there still exists a certain learning effect after operating the job. In such situations, the learning effect is due to the experiences of operating jobs, that is, the total actual processing time of jobs. To the best of our knowledge, apart from the recent paper [29], it has not been investigated the scheduling models considering the actual 
time-dependent learning effect. In this paper, we consider a single machine total completion time minimization problem with past-sequence-dependent setup times and learning effect where the actual processing time of a job is not only an exponential function of the total actual processing times of the jobs already processed but also a function of the job's scheduled position.

\section{Problem Formulation}

The model is described as follows. There are given a single machine and $n$ independent and nonpreemptive jobs that are immediately available for processing. The normal processing time of job $J_{j}$ is $p_{j}(1 \leq j \leq n)$. Associated with each job $J_{j}(1 \leq j \leq n)$ is a weight $w_{j}$ and a due-date $d_{j}$. Let $p_{[k]}$ and $p_{[k]}^{A}$ be the normal processing time and the actual processing time of a job when it is scheduled in the $k$ th position in a sequence, respectively. Let $p_{j r}^{A}$ be the actual processing time of job $J_{j}$ when it is scheduled in position $r$ in a sequence. Then

$$
p_{j r}^{A}=p_{j} \alpha^{-\beta\left(\sum_{k=1}^{r-1} p_{[k]}^{A} / \sum_{k=1}^{n} p_{[k]}\right)} r^{\tau}
$$

where $p_{[1]}^{A}=p_{[1]}, p_{[s]}^{A}=p_{[s]} \alpha^{-\beta\left(\sum_{k=1}^{s-1} p_{[k]}^{A} / \sum_{k=1}^{n} p_{[k]}\right)} s^{\tau}(1<s \leq r-1), \alpha \geq 1, \beta \geq 0$, and $\tau \leq 0$. For convenience, we denote this actual time-dependent and job-position dependent learning effect defined in (2.1) by $L E_{a t-p}$.

As in $[24,26]$, we assume that the p-s-d setup time of a job if it is scheduled in position $r$ is given by

$$
S_{[1]}=0, \quad S_{[r]}=\sum_{k=1}^{r-1} b p_{[k]}^{A},
$$

where $b \geq 0$ is a normalizing constant, and we denote the p-s-d setup given in (2.2) by $s_{p s d}$.

For a given schedule $S$, let $C_{j}(S)$ denote the completion time of job $J_{j}$ and $C_{[r]}(S)$ represent the complete time of the job scheduled in the $r$ th position in $\pi$. For single-machine scheduling with past-sequence-dependent setup times and time-dependent learning effect, Wang in [26] showed that the completion time of the $r$ th job in a given sequence $S$ is given by

$$
C_{[r]}(S)=\sum_{k=1}^{r}(b(r-k)+1) p_{[k]}^{A} .
$$

Similarly, the completion time of the $r$ th job in a given sequence $S$ under (2.1) is

$$
C_{[r]}(S)=\sum_{k=1}^{r}(b(r-k)+1) p_{[k]} \alpha^{-\beta\left(\sum_{l=1}^{k-1} p_{[l]}^{A} / \sum_{l=1}^{n} p_{[l]}\right)} k^{\tau} .
$$

Let $C_{\max }$ denote the makespan, where $C_{\max }=\max \left\{C_{1}, C_{2}, \ldots, C_{n}\right\}$. Let $\sum w_{j} C_{j}$ represent the weighted sum of completion times. In the remaining part of the paper, the problem will be denoted using the three-field notation scheme $1 / L E_{a t-p}, s_{p s d} / \sum w_{j} C_{j}$ introduced by Graham et al. in [30]. 


\section{Total Completion Time Minimization Problem}

In this section, we consider the total completion time minimization problems with pastsequence-dependent setup times defined in (2.2) and the learning effect defined in (2.1).

First, we give three lemmas, which are useful for the following theorem.

Lemma 3.1. It holds that, $t^{\lambda}((r+1) / r)^{\tau}-\lambda t((r+1) / r)^{\tau}+\lambda-1>0$ for $\lambda>1,0 \leq t \leq 1, \tau \leq$ 0 , and $r=1,2, \ldots, n-1$.

Proof. Let

$$
F(t)=t^{\lambda}\left(\frac{r+1}{r}\right)^{\tau}-\lambda t\left(\frac{r+1}{r}\right)^{\tau}+\lambda-1
$$

Taking the first derivative of $F(t)$ with respect to $t$, we have

$$
F^{\prime}(t)=\lambda t^{\lambda-1}\left(\frac{r+1}{r}\right)^{\tau}-\lambda\left(\frac{r+1}{r}\right)^{\tau}=\lambda\left(\frac{r+1}{r}\right)^{\tau}\left(t^{\lambda-1}-1\right)
$$

Since $\lambda>1$ and $0 \leq t \leq 1$, we have $F^{\prime}(t) \leq 0$. This implies that $F(t)$ is nonincreasing on $0 \leq t \leq 1$. Hence,

$$
F(t) \geq F(1)=\left(\frac{r+1}{r}\right)^{\tau}-\lambda\left(\frac{r+1}{r}\right)^{\tau}+\lambda-1=(\lambda-1)\left(1-\left(\frac{r+1}{r}\right)^{\tau}\right)>0
$$

for $\lambda>1, \tau \leq 0$ and $r=1,2, \ldots, n-1$. This completes the proof.

Lemma 3.2. It holds that, $1-\lambda_{1} t((r+1) / r)^{\tau}+\lambda_{2} t \ln t((r+1) / r)^{\tau} \geq 0$ for $0<t \leq 1, \tau \leq 0,0 \leq$ $\lambda_{1} \leq \lambda_{2} \leq 1$, and $r=1,2, \ldots, n-1$.

Proof. Let

$$
F\left(t, \lambda_{1}, \lambda_{2}\right)=1-\lambda_{1} t\left(\frac{r+1}{r}\right)^{\tau}+\lambda_{2} t \ln t\left(\frac{r+1}{r}\right)^{\tau} .
$$

Then $\partial F / \partial \lambda_{1}=-t((r+1) / r)^{\tau}<0, \partial F / \partial \lambda_{2}=t \ln t((r+1) / r)^{\tau} \leq 0$, thus we have

$$
F\left(t, \lambda_{1}, \lambda_{2}\right) \geq F(t, 1,1)=1-t\left(\frac{r+1}{r}\right)^{\tau}+t \ln t\left(\frac{r+1}{r}\right)^{\tau} .
$$

Let

$$
f(t)=1-t\left(\frac{r+1}{r}\right)^{\tau}+t \ln t\left(\frac{r+1}{r}\right)^{\tau} .
$$

Then $f^{\prime}(t)=((r+1) / r)^{\tau} \ln t \leq 0$, hence $f(t)$ is nonijncreasing on $t$ and $f(t) \geq f(1)=1-$ $((r+1) / r)^{\tau} \geq 0$ for $0<t \leq 1$. Therefore, $F\left(t, \lambda_{1}, \lambda_{2}\right) \geq 0$ for $0<t \leq 1, \tau \leq 0,0 \leq \lambda_{1} \leq \lambda_{2} \leq 1$, and $r=1,2, \ldots, n-1$. This completes the proof. 
Lemma 3.3. It holds that, $\mu\left[1-\lambda_{1} t((r+1) / r)^{\tau}\right]-\left[1-\lambda_{2} t^{\mu}((r+1) / r)^{\tau}\right] \geq 0$ for $\mu \geq 1,0<t \leq$ $1, \tau \leq 1,0 \leq \lambda_{1} \leq \lambda_{2} \leq 1$, and $r=1,2, \ldots, n-1$.

Proof. Let

$$
h(\mu)=\mu\left[1-\lambda_{1} t\left(\frac{r+1}{r}\right)^{\tau}\right]-\left[1-\lambda_{2} t^{\mu}\left(\frac{r+1}{r}\right)^{\tau}\right]
$$

To take the first and second derivatives of (3.7) with respect to $\mu$, we have

$$
\begin{gathered}
h^{\prime}(\mu)=1-\lambda_{1} t\left(\frac{r+1}{r}\right)^{\tau}+\lambda_{2} t^{\mu} \ln t\left(\frac{r+1}{r}\right)^{\tau}, \\
h^{\prime \prime \prime}(\mu)=\lambda_{2} t^{\mu} \ln ^{2} t\left(\frac{r+1}{r}\right)^{\tau} \geq 0 .
\end{gathered}
$$

Hence, $h^{\prime}(\mu)$ is nondecreasing on $\mu \geq 1$, that is, $h^{\prime}(\mu) \geq h^{\prime}(1)$ for $\mu \geq 1$. By Lemma 3.2, we have

$$
h^{\prime}(1)=1-\lambda_{1} t\left(\frac{r+1}{r}\right)^{\tau}+\lambda_{2} t \ln t\left(\frac{r+1}{r}\right)^{\tau} \geq 0 .
$$

Therefore, $h^{\prime}(\mu) \geq h^{\prime}(1) \geq 0$. Hence, $h(\mu)$ is nondecreasing on $\mu$, that is, $h(\mu) \geq h(1)=\left(\lambda_{2}-\right.$ $\left.\lambda_{1}\right) t((r+1) / r)^{\tau} \geq 0$ for $t \geq 0, \tau \leq 0,0 \leq \lambda_{1} \leq \lambda_{2} \leq 1$ and $r=1,2, \ldots, n-1$. This completes the proof.

Theorem 3.4. For the total completion time minimization problem $1 / L E_{a t-p}, s_{p s d} / \sum w_{j} C_{j}$ when $p_{j r}^{A}$ is given by (2.1), an optimal schedule can be obtained by sequencing jobs in the WSPT rule if $p_{l} \leq\left((b+1) / 3^{\tau} \beta \ln \alpha\right) \sum_{k=1}^{n} p_{k}(l=1, \ldots, n)$ and jobs have reversely agreeable weights, that is, $p_{i}<p_{j}$ implies $w_{i} \geq w_{j}$ for all jobs $J_{i}$ and $J_{j}$.

Proof (by pair-wise interchanging technique). Consider an optimal schedule $S$ that does not follow the WSPT rule. Then in this schedule there must be at least two adjacent jobs, say job $J_{j}$ followed by job $J_{i}$ such that $p_{j} / w_{j}>p_{i} / w_{i}$ which implies that $p_{j} \geq p_{i}$ by reversely agreeable weights. If $p_{j}>p_{i}$, then $w_{i} \geq w_{j}$; if $p_{i}=p_{j}$, then $w_{i}>w_{j}$. Assume that job $J_{j}$ is scheduled in position $r$. By performing an adjacent pair-wise interchange of jobs $J_{j}$ and $J_{i}$ and remaining all the other jobs in their original positions, we can obtain a new schedule, denoted by $S^{\prime}$. Whereas under the original schedule $J_{j}$ is scheduled in position $r$ and $J_{i}$ is scheduled in position $r+1$, under $S^{\prime}, J_{i}$ is scheduled in position $r$, and $J_{j}$ is scheduled in position $r+1$. Denote $S=\left(\sigma_{1}, J_{j}, J_{i}, \sigma_{2}\right)$, let $C_{\sigma_{1}}$ be the completion time of the last job in $\sigma_{1}$, and let $J_{k}$ be the first job in $\sigma_{2}$.

The completion times of the jobs processed before jobs $J_{j}$ and $J_{i}$ are not affected by the interchange. In the following we will show that $w_{i} C_{i}\left(S^{\prime}\right)+w_{j} C_{j}\left(S^{\prime}\right) \leq w_{i} C_{i}(S)+w_{j} C_{j}(S)$ 
and the completion times of the jobs processed after jobs $J_{j}$ and $J_{i}$ are not increased by the interchanging argument. Under $S$, the completion times of jobs $J_{j}$ and $J_{i}$ are

$$
\begin{gathered}
C_{j}(S)=\sum_{k=1}^{r-1}(b(r-k)+1) p_{[k]}^{A}+p_{j} \alpha^{-\beta\left(\sum_{k=1}^{r-1} p_{[k]}^{A} / \sum_{k=1}^{n} p_{[k]}\right)} r^{\tau}, \\
C_{i}(S)=\sum_{k=1}^{r-1}(b(r+1-k)+1) p_{[k]}^{A}+(b+1) p_{j} \alpha^{-\beta\left(\sum_{k=1}^{r-1} p_{[k]}^{A} / \sum_{k=1}^{n} p_{[k]}\right)} r^{\tau}+p_{i} \alpha^{-\beta\left(\left(\sum_{k=1}^{r-1} p_{[k]}^{A}+p_{j r}^{A}\right) / \sum_{k=1}^{n} p_{[k]}\right)}(r+1)^{\tau}
\end{gathered}
$$

whereas under $S^{\prime}$, they are

$$
\begin{gathered}
C_{i}\left(S^{\prime}\right)=\sum_{k=1}^{r-1}(b(r-k)+1) p_{[k]}^{A}+p_{i} \alpha^{-\beta\left(\sum_{k=1}^{r-1} p_{[k]}^{A} / \sum_{k=1}^{n} p_{[k]}\right)} r^{\tau}, \\
C_{j}\left(S^{\prime}\right)=\sum_{k=1}^{r-1}(b(r+1-k)+1) p_{[k]}^{A}+(b+1) p_{i} \alpha^{-\beta\left(\sum_{k=1}^{r-1} p_{[k]}^{A} / \sum_{k=1}^{n} p_{[k]}\right)} r^{\tau}+p_{j} \alpha^{-\beta\left(\left(\sum_{k=1}^{r-1} p_{[k]}^{A}+p_{j r}^{A}\right) / \sum_{k=1}^{n} p_{[k]}\right)}(r+1)^{\tau} .
\end{gathered}
$$

So we have

$$
\begin{aligned}
& w_{i} C_{i}(S)+w_{j} C_{j}(S)-w_{i} C_{i}\left(S^{\prime}\right)-w_{j} C_{j}\left(S^{\prime}\right) \\
&= b\left(w_{i}-w_{j}\right) \sum_{k=1}^{r-1} p_{[k]}^{A}+b\left(w_{i} p_{j}-w_{j} p_{i}\right) \alpha^{-\beta\left(\sum_{k=1}^{r-1} p_{[k]}^{A} / \sum_{k=1}^{n} p_{[k]}\right)} r^{\tau} \\
&+\left(w_{i}+w_{j}\right)\left(p_{j}-p_{i}\right) \alpha^{-\beta\left(\sum_{k=1}^{r-1} p_{[k]}^{A} / \sum_{k=1}^{n} p_{[k]}\right)} r^{\tau} \\
&+w_{i} p_{i} \alpha^{-\beta\left(\left(\sum_{k=1}^{r-1} p_{[k]}^{A}+p_{j r}^{A}\right) / \sum_{k=1}^{n} p_{[k]}\right)}(r+1)^{\tau}-w_{j} p_{j} \alpha^{-\beta\left(\left(\sum_{k=1}^{r-1} p_{[k]}^{A}+p_{i r}^{A}\right) / \sum_{k=1}^{n} p_{[k]}\right)}(r+1)^{\tau} .
\end{aligned}
$$

Let $x=\alpha^{-\beta\left(\sum_{k=1}^{r-1} p_{[k]}^{A} / \sum_{k=1}^{n} p_{[k]}\right)}, \lambda_{1}=w_{j} /\left(w_{i}+w_{j}\right), \lambda_{2}=w_{i} /\left(w_{i}+w_{j}\right), \mu=p_{j} / p_{i}$, and $t=\alpha^{-\beta\left(p_{i r}^{A} / \sum_{k=1}^{n} p_{k}\right)}$. Clearly $\mu \geq 1,0 \leq \lambda_{1} \leq \lambda_{2} \leq 1$, and $t>0$. Then the last three terms in (3.12) can be rewritten as

$$
\begin{aligned}
& w_{i} C_{i}(S)+w_{j} C_{j}(S)-w_{i} C_{i}\left(S^{\prime}\right)-w_{j} C_{j}\left(S^{\prime}\right) \\
& \quad=p_{i}\left(w_{i}+w_{j}\right) r^{\tau} x\left\{\mu\left[1-\lambda_{1} t\left(\frac{r+1}{r}\right)^{\tau}\right]-\left[1-\lambda_{2} t^{\mu}\left(\frac{r+1}{r}\right)^{\tau}\right]\right\}
\end{aligned}
$$

$$
\geq 0 \text { (by Lemma 3.3). }
$$

From $p_{j} / w_{j}>p_{i} / w_{i}$, we have $b\left(w_{i} p_{j}-w_{j} p_{i}\right) \alpha^{-\beta\left(\sum_{k=1}^{r-1} p_{[k]}^{A} / \sum_{k=1}^{n} p_{[k]}\right)} r^{\tau}>0$. In addition, from $p_{j} \geq p_{i}$, which implies that $w_{i} \geq w_{j}$, we have $b\left(w_{i}-w_{j}\right) \sum_{k=1}^{r-1} p_{[k]}^{A} \geq 0$. Hence,

$$
w_{i} C_{i}(S)+w_{j} C_{j}(S)>w_{i} C_{i}\left(S^{\prime}\right)+w_{j} C_{j}\left(S^{\prime}\right)
$$


Next, we will show that the completion times of the jobs in $\sigma_{2}$ are not increased by the interchanging argument. Firstly, we show that $C_{j}\left(S^{\prime}\right) \leq C_{i}(S)$ after interchanging. Since

$$
\frac{C_{j}\left(S^{\prime}\right)-C_{i}(S)}{p_{i} x r^{\tau}}=(b+1)\left(1-\frac{p_{j}}{p_{i}}\right)+\frac{p_{j}}{p_{i}} \alpha^{-\beta\left(p_{i r}^{A} / \sum_{k=1}^{n} p_{[k]}\right)}\left(\frac{r+1}{r}\right)^{\tau}-\alpha^{-\beta\left(p_{j r}^{A} / \sum_{k=1}^{n} p_{[k]}\right)}\left(\frac{r+1}{r}\right)^{\tau},
$$

then let $t=\alpha^{-\beta\left(p_{i r}^{A} / \sum_{k=1}^{n} p_{k}\right)}$ and $\lambda=p_{j} / p_{i}$. It is clearly that $t \geq 0$ and $\lambda>1$. By substituting $t$ and $\lambda$ into (3.15), it is simplified to

$$
\frac{C_{j}\left(S^{\prime}\right)-C_{i}(S)}{p_{i} x r^{\tau}}=\left\{(b+1)(1-\lambda)+\lambda t\left(\frac{r+1}{r}\right)^{\tau}-t^{\lambda}\left(\frac{r+1}{r}\right)^{\tau}\right\}
$$

By Lemma 3.1, we have $C_{j}\left(S^{\prime}\right)<C_{i}(S)$.

Secondly, we show that the completion times of the jobs in $\sigma_{2}$ are not increased. Note that $J_{k}$ is the first job in $\sigma_{2}$, it is scheduled in $(r+2)$ th position, let

$$
\begin{aligned}
& \Lambda_{1}=p_{i} \alpha^{-\beta\left(\sum_{k=1}^{r-1} p_{[k]}^{A} / \sum_{k=1}^{n} p_{[k]}\right)} r^{\tau}+p_{j} \alpha^{-\beta\left(\sum_{k=1}^{r} p_{[k]}^{A} / \sum_{k=1}^{n} p_{[k]}\right)}(r+1)^{\tau}, \\
& \Lambda_{2}=p_{j} \alpha^{-\beta\left(\sum_{k=1}^{r-1} p_{[k]}^{A} / \sum_{k=1}^{n} p_{[k]}\right)} r^{\tau}+p_{i} \alpha^{-\beta\left(\sum_{k=1}^{r} p_{[k]}^{A} / \sum_{k=1}^{n} p_{[k]}\right)}(r+1)^{\tau} .
\end{aligned}
$$

Then we have

$$
\frac{\Lambda_{1}-\Lambda_{2}}{p_{i} x r^{\tau}}=(1-\lambda)+\lambda t\left(\frac{r+1}{r}\right)^{\tau}-t^{\lambda}\left(\frac{r+1}{r}\right)^{\tau}
$$

By Lemma 3.1 we have $\Lambda_{1}<\Lambda_{2}$. Since

$$
\begin{aligned}
C_{k}\left(S^{\prime}\right)= & C_{j}\left(S^{\prime}\right)+b\left(\sum_{l=1}^{r-1} p_{[l]}^{A}+p_{i r}^{A}+p_{j r+1}^{A}\right)+p_{k} \alpha^{-\beta\left(\left(\sum_{l=1}^{r-1} p_{[l]}^{A}+p_{i r}^{A}+p_{j r+1}^{A}\right) / \sum_{l=1}^{n} p_{[l]}\right)}(r+2)^{\tau} \\
= & \sum_{k=1}^{r-1}(b(r+2-k)+1) p_{[k]}^{A}+(2 b+1) p_{i} \alpha^{-\beta\left(\sum_{k=1}^{r-1} p_{[k]}^{A} / \sum_{k=1}^{n} p_{[k]}\right)} r^{\tau} \\
& +(b+1) p_{j} \alpha^{-\beta\left(\left(\sum_{k=1}^{r-1} p_{[k]}^{A}+p_{i r}^{A}\right) / \sum_{k=1}^{n} p_{[k]}\right)}(r+1)^{\tau}+p_{k} \alpha^{-\beta\left(\left(\sum_{l=1}^{r-1} p_{[l]}^{A}+p_{i r}^{A}+p_{j r+1}^{A}\right) / \sum_{l=1}^{n} p_{[l]}\right)}(r+2)^{\tau}, \\
C_{k}(S)= & C_{i}(S)+b\left(\sum_{l=1}^{r-1} p_{[l]}^{A}+p_{j r}^{A}+p_{i r+1}^{A}\right)+p_{k} \alpha^{-\beta\left(\left(\sum_{l=1}^{r-1} p_{[l]}^{A}+p_{j r}^{A}+p_{i r+1}^{A}\right) / \sum_{l=1}^{n} p_{[l]}\right)}(r+2)^{\tau} \\
= & \sum_{k=1}^{r-1}(b(r+2-k)+1) p_{[k]}^{A}+(2 b+1) p_{j} \alpha^{-\beta\left(\sum_{k=1}^{r-1} p_{[k]}^{A} / \sum_{k=1}^{n} p_{[k]}\right)} r^{\tau} \\
& +(b+1) p_{i} \alpha^{-\beta\left(\left(\sum_{k=1}^{r-1} p_{[k]}^{A}+p_{j r}^{A}\right) / \sum_{k=1}^{n} p_{[k]}\right)}(r+1)^{\tau}+p_{k} \alpha^{-\beta\left(\left(\sum_{l=1}^{r-1} p_{[l]}^{A}+p_{j r}^{A}+p_{i r+1}^{A}\right) / \sum_{l=1}^{n} p_{[l]}\right)}(r+2)^{\tau} .
\end{aligned}
$$


Then we have

$$
\begin{aligned}
C_{k}\left(S^{\prime}\right)-C_{k}(S)= & b\left(p_{i}-p_{j}\right) \alpha^{-\beta\left(\sum_{k=1}^{r-1} p_{[k]}^{A} / \sum_{k=1}^{n} p_{[k]}\right)} r^{\tau}+(b+1)\left(\Lambda_{1}-\Lambda_{2}\right) \\
& +p_{k}(r+2)^{\tau}\left(\alpha^{-\beta\left(\left(\Lambda+\Lambda_{1}\right) / P\right)}-\alpha^{-\beta\left(\left(\Lambda+\Lambda_{2}\right) / P\right)}\right) \\
< & (b+1)\left(\Lambda_{1}-\Lambda_{2}\right)+p_{k}(r+2)^{\tau}\left(-\frac{\beta \ln \alpha}{P} \alpha^{-\beta((\Lambda+\xi) / P)}\right)\left(\Lambda_{1}-\Lambda_{2}\right) \\
= & \left(\Lambda_{2}-\Lambda_{1}\right)\left[p_{k}(r+2)^{\tau}\left(\frac{\beta \ln \alpha}{P} \alpha^{-\beta((\Lambda+\xi) / P)}\right)-(b+1)\right] \\
< & {\left[\left(\beta 3^{\tau} \ln \alpha\right) \frac{p_{k}}{P}-(b+1)\right]\left(\Lambda_{2}-\Lambda_{1}\right) . }
\end{aligned}
$$

For any job $J_{l}$, by assumption $p_{l} \leq(b+1) / 3^{\tau} \beta \ln \alpha \sum_{k=1}^{n} p_{k}$ for $\alpha \geq 1$ and $\beta \geq 0$. Then (3.20) is not larger than zero, that is, $C_{k}\left(S^{\prime}\right) \leq C_{k}(S)$. Similarly, we have $C_{h}\left(S^{\prime}\right) \leq C_{h}(S)$ for any $J_{h}$ in $\sigma_{2}$. the theorem.

Repeating this interchange argument for all jobs not sequenced in the WSPT rule yields

Corollary 3.5. For the problem $1 / L E_{a t-p}, s_{p s d} / C_{\max }$ when $p_{j r}^{A}$ is given by (2.1), if $p_{l} \leq$ $\sum_{k=1}^{n} p_{k} / 3^{\tau} \beta \ln \alpha(l=1, \ldots, n)$, then there exists an optimal schedule in which the job sequence is determined by the SPT rule.

Proof. it can be directly from the proof of Theorem 3.4.

Corollary 3.6. For the problem $1 / L E_{a t-p}, s_{p s d} / \sum C_{j}$ when $p_{j r}^{A}$ is given by (2.1), if $p_{l} \leq$ $\sum_{k=1}^{n} p_{k} / 3^{\tau} \beta \ln \alpha(l=1, \ldots, n)$, then there exists an optimal schedule in which the job sequence is determined by the SPT rule.

Corollary 3.7. For the problem $1 / L E_{a t-p}, s_{p s d} / \sum C_{j}^{k}$ when $p_{j r}^{A}$ is given by (2.1), if $p_{l} \leq$ $\sum_{k=1}^{n} p_{k} / 3^{\tau} \beta \ln \alpha(l=1, \ldots, n)$, then there exists an optimal schedule in which the job sequence is determined by the SPT rule.

Proof. Here, we still use the same notations as in the proof of Theorem 3.4. In order to show $S^{\prime}$ dominates $S$, it suffices to show that (i) $C_{j}\left(S^{\prime}\right) \leq C_{i}(S)$, (ii) $C_{l}\left(S^{\prime}\right) \leq C_{l}(S)$ for any job $J_{l}$ $(l \neq i, j)$ and (iii) $C_{i}^{k}\left(S^{\prime}\right)+C_{j}^{k}\left(S^{\prime}\right) \leq C_{i}^{k}(S)+C_{j}^{k}(S)$.

The proof of part (i) and part (ii) are given in Theorem 3.4. In addition, from $p_{i} \leq p_{j}$, we have $C_{i}\left(S^{\prime}\right) \leq C_{j}(S)$. Since $k$ is a positive real number, we have $C_{i}^{k}\left(S^{\prime}\right)+C_{j}^{k}\left(S^{\prime}\right) \leq C_{i}^{k}(S)+C_{j}^{k}(S)$. This completes the proof.

Corollary 3.8. For the problem $1 / L E_{a t-p}, s_{p s d}, p_{j}=p / \sum w_{j} C_{j}$ when $p_{j r}$ is given by (2.1), an optimal schedule can be obtained by sequencing jobs in non-increasing order of $w_{j}$.

Corollary 3.9. For the problem $1 / L E_{a t-p}, s_{p s d}, w_{j}=k p_{j} / \sum w_{j} C_{j} w$ wen $p_{j r}$ is given by (2.1), if $p_{l} \leq\left((b+1) / 3^{\tau} \beta \ln \alpha\right) \sum_{k=1}^{n} p_{k}(l=1, \ldots, n)$, then an optimal schedule can be obtained by sequencing jobs in SPT rule. 
Remark 3.10. In this paper, the learning effect is defined in (2.1) and p-s-d setup times is defined in (2.2), where the $\alpha \geq 1, \beta \geq 0, \tau \leq 0$, and $b \geq 0$ are controlled parameters. In practises, even human interactions have a significant impact during the processing of jobs, the impact should be limited. In general, half of the decreasing on the processing time is impossible. So the parameters should have practical meaning and properly given. If there parameters satisfy that $\left((b+1) / 3^{\tau} \beta \ln \alpha\right)<1$, then for any job $p_{l}(l=1,2, \ldots, n)$ we have $p_{l} \leq\left((b+1) / 3^{\tau} \beta \ln \alpha\right) \sum_{k=1}^{n} p_{k}$. Thus, Theorem 3.4 holds under agreeable condition.

\section{Conclusions}

In this paper we investigated the single machine total weighted completion time minimization problem with p-s-d setup times and learning effect where the actual processing time of a job is not only an exponential function of the total actual processing times of the jobs already processed but also a function of the job's scheduled position. We show that the classical WSPT rule is optimal under certain conditions. It is suggested for future research to investigate other efficient heuristic algorithm to the problem or extend this kind of learning into the context of other more complicated scheduling settings, or propose more general and practical models.

\section{Acknowledgment}

This research is supported by the National Natural Science Foundation of China (60774049).

\section{References}

[1] A. B. Badiru, "Computational survey of univariate and multivariate learning curve models," IEEE Transactions on Engineering Management, vol. 39, no. 2, pp. 176-188, 1992.

[2] D. Biskup, "Single-machine scheduling with learning considerations," European Journal of Operational Research, vol. 115, no. 1, pp. 173-178, 1999.

[3] T. C. E. Cheng and G. Wang, "Single machine scheduling with learning effect considerations," Annals of Operations Research, vol. 98, no. 1-4, pp. 273-290, 2000.

[4] G. Mosheiov, "Scheduling problems with a learning effect," European Journal of Operational Research, vol. 132, no. 3, pp. 687-693, 2001.

[5] G. Mosheiov, "Parallel machine scheduling with a learning effect," Journal of the Operational Research Society, vol. 52, no. 10, pp. 1165-1169, 2001.

[6] G. Mosheiov and J. B. Sidney, "Scheduling with general job-dependent learning curves," European Journal of Operational Research, vol. 147, no. 3, pp. 665-670, 2003.

[7] A. Bachman and A. Janiak, "Scheduling jobs with position-dependentprocessing times," Journal of the Operational Research Society, vol. 55, no. 3, pp. 257-264, 2004.

[8] J.-B. Wang, "A note on scheduling problems with learning effects and deteriorating jobs," International Journal of Systems Science, vol. 37, no. 12, pp. 827-833, 2006.

[9] J.-B. Wang, "Single-machine scheduling problems with the effects of learning and deterioration," Omega, vol. 35, no. 4, pp. 397-402, 2007.

[10] T. Eren and E. Güner, "Minimizing total tardiness in a scheduling problem with a learning effect," Applied Mathematical Modelling, vol. 31, no. 7, pp. 1351-1361, 2007.

[11] B. M. T. Lin, "Complexity results for single-machine scheduling with positional learning effects," Journal of the Operational Research Society, vol. 58, no. 8, pp. 1099-1102, 2007.

[12] X. Wang and T. C. E. Cheng, "Single-machine scheduling with deteriorating jobs and learning effects to minimize the makespan," European Journal of Operational Research, vol. 178, no. 1, pp. 57-70, 2007.

[13] C.-C. Wu, W.-C. Lee, and T. Chen, "Heuristic algorithms for solving the maximum lateness scheduling problem with learning considerations," Computers $\mathcal{E}$ Industrial Engineering, vol. 52, no. 1, pp. 124-132, 2007. 
[14] W.-H. Kuo and D.-L. Yang, "Minimizing the total completion time in a single-machine scheduling problem with a time-dependent learning effect," European Journal of Operational Research, vol. 174, no. 2, pp. 1184-1190, 2006.

[15] W.-H. Kuo and D.-L. Yang, "Single-machine group scheduling with a time-dependent learning effect," Computers E Operations Research, vol. 33, no. 8, pp. 2099-2112, 2006.

[16] W.-H. Kuo and D.-L. Yang, "Single-machine scheduling problems with time-dependent learning effect," Computers \& Mathematics with Applications, vol. 53, no. 11, pp. 1733-1739, 2007.

[17] J.-B. Wang, C. T. Ng, T. C. E. Cheng, and L. L. Liu, "Single-machine scheduling with a time-dependent learning effect," International Journal of Production Economics, vol. 111, no. 2, pp. 802-811, 2008.

[18] C. Koulamas and G. J. Kyparisis, "Single-machine and two-machine flowshop scheduling with general learning functions," European Journal of Operational Research, vol. 178, no. 2, pp. 402-407, 2007.

[19] C.-C. Wu and W.-C. Lee, "Single-machine scheduling problems with a learning effect," Applied Mathematical Modelling, vol. 32, no. 7, pp. 1191-1197, 2008.

[20] T. C. E. Cheng, C.-C. Wu, and W.-C. Lee, "Some scheduling problems with sum-of-processing-timesbased and job-position-based learning effects," Information Sciences, vol. 178, no. 11, pp. 2476-2487, 2008.

[21] D. Biskup, "A state-of-the-art review on scheduling with learning effects," European Journal of Operational Research, vol. 188, no. 2, pp. 315-329, 2008.

[22] A. Allahverdi, J. N. D. Gupta, and T. Aldowaisan, "A review of scheduling research involving setup considerations," Omega, vol. 27, no. 2, pp. 219-239, 1999.

[23] A. Allahverdi, C. T. Ng, T. C. E. Cheng, and M. Y. Kovalyov, "A survey of scheduling problems with setup times or costs," European Journal of Operational Research, vol. 187, no. 3, pp. 985-1032, 2008.

[24] C. Koulamas and G. J. Kyparisis, "Single-machine scheduling problems with past-sequencedependent setup times," European Journal of Operational Research, vol. 187, no. 3, pp. 1045-1049, 2008.

[25] W.-H. Kuo and D.-L. Yang, "Single machine scheduling with past-sequence-dependent setup times and learning effects," Information Processing Letters, vol. 102, no. 1, pp. 22-26, 2007.

[26] J.-B. Wang, "Single-machine scheduling with past-sequence-dependent setup times and timedependent learning effect," Computers E Industrial Engineering, vol. 55, no. 3, pp. 584-591, 2008.

[27] D. Biskup and J. Herrmann, "Single-machine scheduling against due dates with past-sequencedependent setup times," European Journal of Operational Research, vol. 191, no. 2, pp. 586-591, 2008.

[28] J.-B. Wang, D. Wang, L.-Y. Wang, L. Lin, N. Yin, and W.-W. Wang, "Single machine scheduling with exponential time-dependent learning effect and past-sequence-dependent setup times," Computers $\mathcal{E}$ Mathematics with Applications, vol. 57, no. 1, pp. 9-16, 2009.

[29] D.-L. Yang and W.-H. Kuo, "Single-machine scheduling with an actual time-dependent learning effect," Journal of the Operational Research Society, vol. 58, no. 10, pp. 1348-1353, 2007.

[30] R. L. Graham, E. L. Lawler, J. K. Lenstra, and A. H. G. Rinnooy Kan, “Optimization and approximation in deterministic sequencing and scheduling: a survey," Annals of Discrete Mathematics, vol. 5, pp. 287326, 1979. 


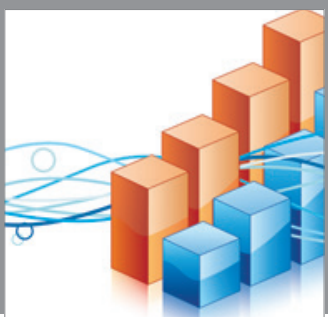

Advances in

Operations Research

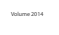

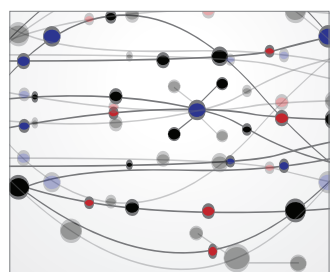

\section{The Scientific} World Journal
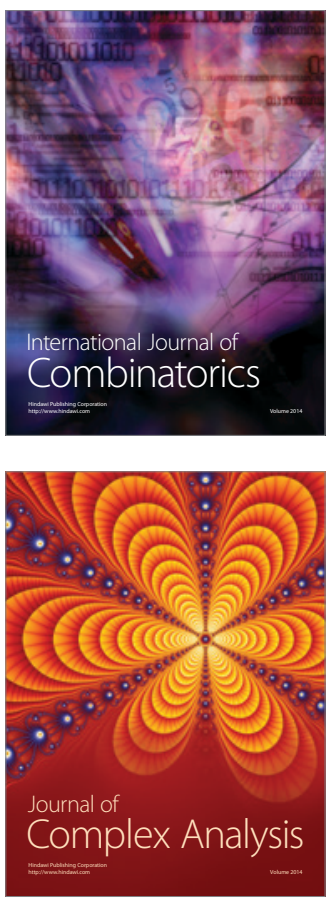

International Journal of

Mathematics and

Mathematical

Sciences
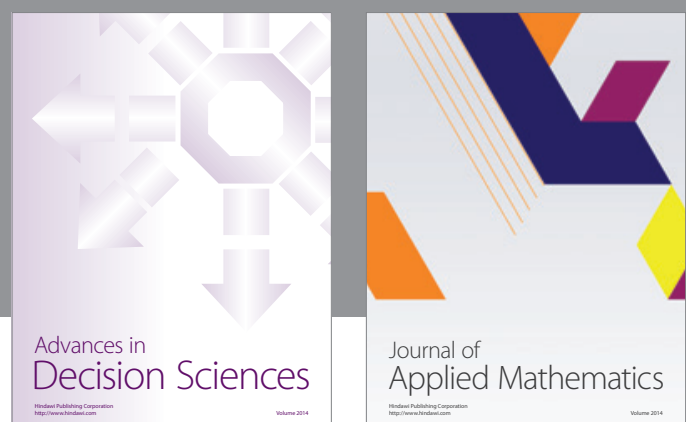

Journal of

Applied Mathematics
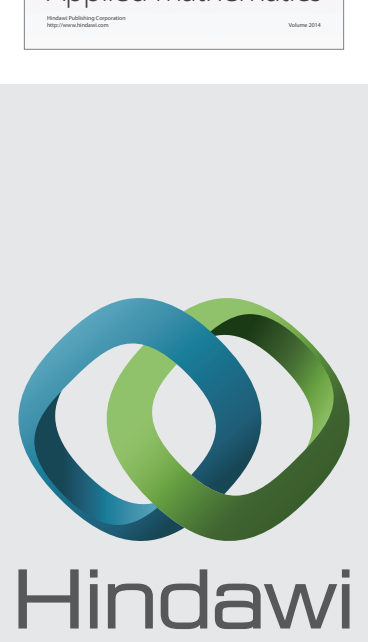

Submit your manuscripts at http://www.hindawi.com
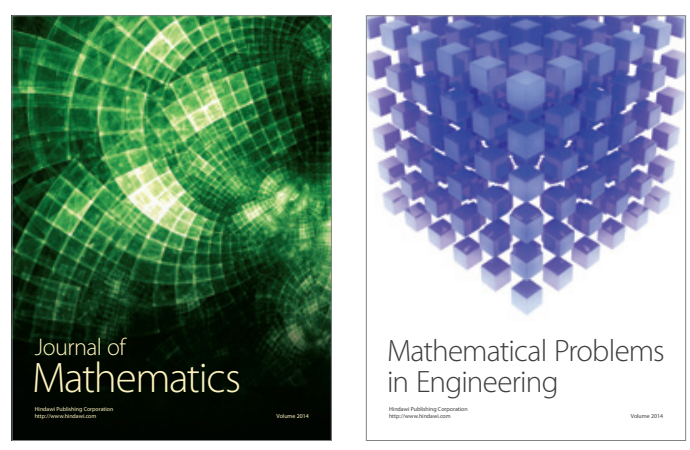

Mathematical Problems in Engineering
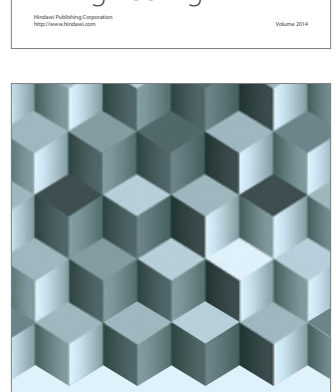

Journal of

Function Spaces


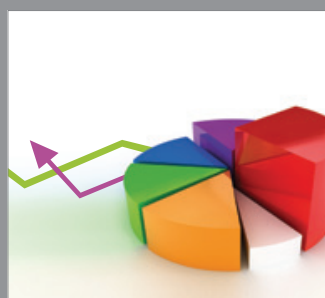

ournal of

Probability and Statistics

Promensencen
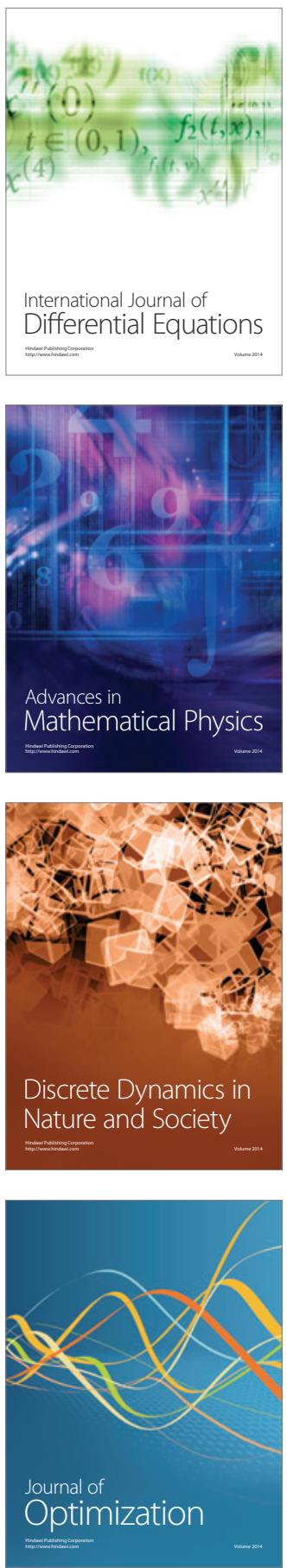